\title{
Comparison of NEAT and Backpropagation Neural Network on Breast Cancer Diagnosis
}

\author{
Hamza Turabieh \\ Information Technology Department, CIT Collage \\ Taif University, KSA
}

\begin{abstract}
In this paper we present a comparison between NeuroEvolution of Augmenting Typologies (NEAT) algorithm with Backpropagation Neural Network for the prediction of breast cancer. Machine learning algorithms could be used to enhance the performance of medical practitioners in the diagnosis of breast cancer. NEAT is a promising machine learning algorithm, which combines genetic algorithms and neural network. We compare the performance of these two algorithms on a standard benchmark dataset. Our results demonstrate that NEAT outperforms Backpropagation Neural Network, and we show that experimentally that NEAT has better generalization and much lower computational cost.
\end{abstract}

\section{Keywords}

NEAT, Backpropagation, Breast Cancer.

\section{INTRODUCTION}

Breast cancer is one of the most common cancers in women. According to the World Health Organization (WHO), 1.5 million people die from breast cancer each year. Breast cancer is considering one of the top cancers diagnosed, which cause death among women. Generally, breast cancer does not produce symptoms until they are relatively large at advance stage.

The economic and social values of Breast Cancer Diagnosis (BCD) are very high. As a result, research communities form the fields of computational intelligence and machine learning have invested the broad research spectrum of building algorithms for achieving highly accurate classification over the years [1][2][3]. Artificial Neural Networks (ANN) is one of the most famous methods for classification problems. The researchers applied ANN mostly to solve BCD problem, which is consider the most common method. The main objective of ANN is to support doctors with their jobs, which tries to provide a filter that distinguishes the cases that do not have cancer, in order to reduce the cost of medication treatment. The results comes from ANN can be used to reduce the disagreement and inconsistencies in mammographic interpretation. In medical field, ANN has been applied in different area as shown in Figure 1. However, to use ANN in clinic, we have to build a model that is able to provide accurate and reliable results for diagnosis the breast cancer and all the obtained results are confirmed by a human doctor.

Huge amount of distinguished papers have been published for predicting breast cancer. Rumelhart et al. [4] applied ANN that was trained based on Backpropagation algorithm, the structure of the proposed ANN is 10 hidden nodes and one single output node was trained to give 0 for benign and 1 for malignancy. The authors report that the performance of ANN is competitive to the domain expert. The performance is enhanced and out performs the domain of expert after considering amount of feature selection. Wu et al. [5] applied
ANN to learn 133 samples containing each 43 mammographic features rated between $0-10$ by a mammographer. Wilding et al. [6] applied Backpropagation method, where the input sets derived from a group of blood tests. However, the performance of the proposed ANN failed to perform well.

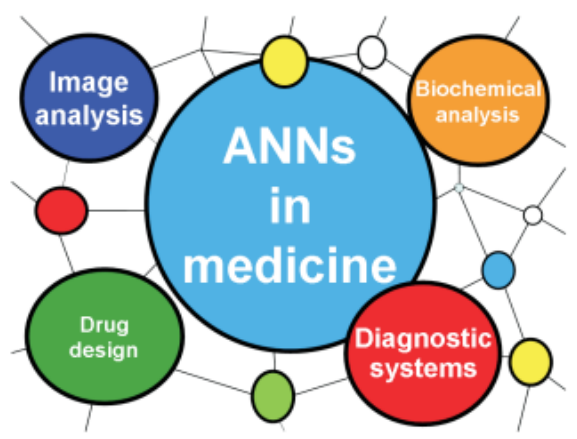

Fig 1: The main applications of ANN in medicine. [16]

Floyd et al. [7] applied Backpropagation method, which used eight input parameters; these are margin, mass size, asymmetric density, architectural distortion, classification number, morphology, density and distribution. The authors applied their experiments over limited data sets of 260 cases, and the accuracy of their ANN is 50\%. In general, Backpropagation disadvantage is easily trapped in local minimum. Therefore, Fogel et al. [8] applied evolutionary programming to train ANN to overcome the disadvantage of Backpropagation.

Setiono [9] applied ANN based on backpropagation in training process and achieved an accuracy over tested dataset about $94 \%$. The author applied his rules to extract useful rules that are able to predict breast cancer. However, the accuracy of the extracted rules did not make difference. Setiono improve his work based on feature selection before start training process [10]. The new approach increases the accuracy of the previous method about $2 \%$. However Setiono results are comparable with Fogel et al. [8].

Pendharkar et al. [11] applied ANN and data envelopment analysis for mining breast cancer. They found that ANN performance is better than data envelopment analysis algorithm. Furundzic et al. [12] applied ANN based on Backpropagation. The authors start with 47 inputs and 5 hidden nodes. The simulations results are performed over 200 instances and the accuracy is $95 \%$. However, after significant analysis and feature selection, they reduce the input to 29 and the accuracy result not change.

Jong et al. [13] applied hybrid Bayesian network and standard ANN to predict breast cancer. The accuracy of the proposed hybrid is $87.2 \%$, while the accuracy of the standard ANN is $88.8 \%$. There are several research papers that 
investigate breast cancer prediction, interested readers can found in [14] [15].

In general, ANN has been confirmed as a robust method for breast cancer diagnosis [7][8], which is consider as superior to conventional methods that is applied for breast cancer prediction such as tumor, Node, Metastasis (TNM) staging system and logistic regression [9]. The main reason behind using ANN method its ability in capturing the complex and nonlinear interaction between prognostic makers and the outcome that should be predicted.

This paper compares the performance of NeuroEvolution of Augmenting Topologies (NEAT) algorithm with Backpropagation algorithm (BP) to predict breast cancer. The remainder of the paper is organized as follows: Section 2 explains depicts the benchmark datasets for BCD. Section 3 illustrates the NEAT and Backpropagation methods. Experimental results are discussed in Section 4, followed by some brief concluding comments in Section 5.

\section{PROBLEM DISCRIBTION}

The Wisconsin Breast Cancer datasets from the UCI Machine Learning Repository is used [16], The class output includes 2 classes, benign and malignant. This dataset contains 699 patterns with 9 attributes; clump thickness, uniformity of cell size, uniformity of cell shape, marginal adhesion, single eptihelail cell size, bare nuclei, bland chromatin, normal nucleoli, and mistoses. A brief description of these attributes is presented in Table I.

\section{THE ALGORITHM}

In this section, NEAT algorithm first described. Then, backpropagation neural network is introduced in detail.

\subsection{NEAT Algorithm}

NEAT (NeuroEvolution of Augmenting Topologies) algorithm is a neuroEvolution developed at the University of Texas at Austin 2002 [17]. This is one of the most popular constructive neural network algorithms. It has an input and output layer, just like the more common feedforward neural networks. A NEAT network starts with only an input layer and output layer, which use complexification to search through the high-dimensional space of network topologies, the size of neural network is increase only if it is helpful in finding a better and more complex controllers than standard neural network. Since NEAT starts searching in relatively low-dimensional space, it avoids wasting time looking for evolving neural networks complex tasks.

NEAT encodes neural network structure and connection weights using nodes, and uses the biological principles of increasing complexity and speciation. The main structure of NEAT genotype has two lists which they are; a list of neuron genes and a list of link genes. The main task of neuron genes assign a neuron an identification number and indicate whether it is an input, output, or hidden node. While link genes contain information about two connected neurons (nodes), and links are weighted connections. As standard neural networks, these weights will be adjusted. While the neural network learns, NEAT is able to adjust both links and weights at the same time. NEAT links structure has three categories which they are: Forward Link: A connection from one neuron to another neuron further down the neural network. Recurrent Link: A connection from one neuron to an earlier neuron in the neural network, and Self-Connected: A connection from one neuron to itself. Figure 2 illustrates these categories of connection used in NEAT structure.

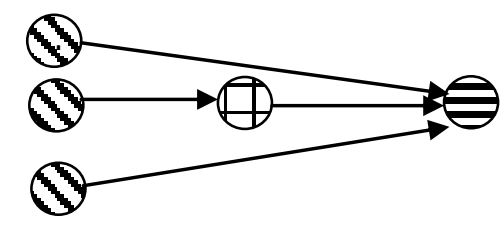

a) Forward Link
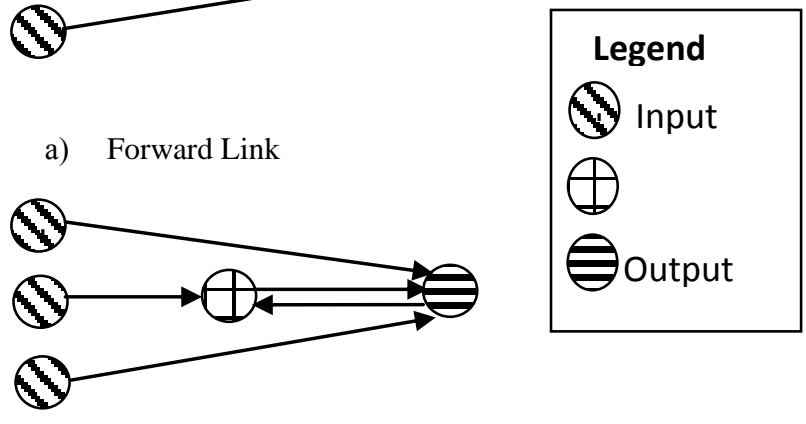

b) Recurrent Links

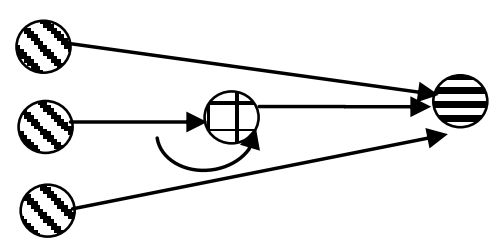

c) Self-Connected links

\section{Fig. 2: a) Forward link, b) Recurrent links and c) Self} connected links

Moreover, there are three fundamental challenges in evolving neural network topology, Table 2 illustrates these challenges as questions and answers that motivate to use NEAT algorithm. Figure 3 demonstrate a NEAT genotype mapping example. There are three input nodes, one hidden node, one output node and seven connection definitions, one of which is recurrent. The second gene is disabled, so the connection that it specifies (between node 2 and 4 ) is not expressed in the phenotype. The genptype can have arbitrary length, and thereby represent arbitrarily complex networks. Innovation numbers, which allow NEAT to identify which gens matchup between different genomes, are shown on top of each gene. This encoding is efficient and allows changes the network structure during evaluation. 
Table 1: Breast Cancer Wisconsin dataset attributes

\begin{tabular}{|c|c|c|}
\hline Attribute & Domain & Description \\
\hline Clump thickness & $1-10$ & $\begin{array}{l}\text { The benign cells tend to be grouped in monolayers, while malignant cells are } \\
\text { often grouped in multilayer. }\end{array}$ \\
\hline Uniformity of cell size & $1-10$ & \multirow{2}{*}{$\begin{array}{l}\text { The malignant cells tend to vary in size and shape. That is why these parameters } \\
\text { are valuable in determining whether the cells are cancerous or not. }\end{array}$} \\
\hline Uniformity of cell shape & $1-10$ & \\
\hline Marginal adhesion & $1-10$ & $\begin{array}{l}\text { The normal cells tend to stick together. Where cancer cells tend to lose this } \\
\text { ability. So loss of adhesion is a sign of malignancy. }\end{array}$ \\
\hline Single eptihelail cell size & $1-10$ & $\begin{array}{l}\text { In the Single epithelial cell size, where the size is related to the uniformity } \\
\text { mentioned above. }\end{array}$ \\
\hline Bare nuclei & $1-10$ & $\begin{array}{l}\text { The Bare nuclei is a term used for nuclei that is not surrounded by cytoplasm (the } \\
\text { rest of the cell). Those are typically seen in benign tumors. }\end{array}$ \\
\hline Bland chromatin & $1-10$ & $\begin{array}{l}\text { The Bland Chromatin describes a uniform "texture" of the nucleus seen in benign } \\
\text { cells. In cancer cells the chromatin tends to be coarser. }\end{array}$ \\
\hline Normal nucleoli & $1-10$ & $\begin{array}{l}\text { In normal cells the nucleolus is usually very small if visible. In cancer cells the } \\
\text { nucleoli become more prominent, and sometimes there are more of them. }\end{array}$ \\
\hline Mistoses & $1-10$ & $\begin{array}{l}\text { Mitosis is nuclear division plus cytokines and produce two identical daughter } \\
\text { cells during prophase. It is the process in which the cell divides and replicates. } \\
\text { Pathologists can determine the grade of cancer by counting the number of } \\
\text { mitoses. }\end{array}$ \\
\hline
\end{tabular}

Table 2: Challenges and answers in evolving neural network topology

\begin{tabular}{|c|l|l|}
\hline Challenges & Questions & Answer \\
\hline 1 & $\begin{array}{l}\text { What kind of genetic representation would } \\
\text { allow disparate to topologies to crossover in } \\
\text { the meaningful way? }\end{array}$ & $\begin{array}{l}\text { Use historical marking to line up genes with the same } \\
\text { origin }\end{array}$ \\
\hline 2 & $\begin{array}{l}\text { How can topological innovation that needs } \\
\text { a few generations to optimize be protected } \\
\text { so that it does not disappear from the } \\
\text { population prematurely? }\end{array}$ & Separate each innovation into different species. \\
\hline 3 & $\begin{array}{l}\text { How can topology minimized through } \\
\text { evolution so the most efficient solutions } \\
\text { will be discovered? }\end{array}$ & $\begin{array}{l}\text { Start from minimal structure and add nodes and } \\
\text { connections incrementally. }\end{array}$ \\
\hline
\end{tabular}

\begin{tabular}{|c|c|c|c|c|c|c|c|}
\hline \multicolumn{8}{|l|}{ Genome (Genotype) } \\
\hline Node Genes & $\begin{array}{l}\text { Node1 } \\
\text { Input }\end{array}$ & $\begin{array}{l}\text { Node2 } \\
\text { Input }\end{array}$ & $\begin{array}{l}\text { Node3 } \\
\text { Input }\end{array}$ & $\begin{array}{l}\text { Node4 } \\
\text { Output }\end{array}$ & $\begin{array}{l}\text { Node5 } \\
\text { Hidden }\end{array}$ & & \\
\hline Connect Genes & $\begin{array}{l}\text { Innov1 } \\
\text { In } 1 \\
\text { Out } 4 \\
\text { Weight } 0.7 \\
\text { Enabled }\end{array}$ & $\begin{array}{l}\text { Innov } 2 \\
\text { In } 2 \\
\text { Out } 4 \\
\text { Weight -0.5 } \\
\text { Disabled }\end{array}$ & $\begin{array}{l}\text { Innov } 3 \\
\text { In } 3 \\
\text { Out } 4 \\
\text { Weight } 0.7 \\
\text { Enabled }\end{array}$ & $\begin{array}{l}\text { Innov } 4 \\
\text { In } 3 \\
\text { Out } 5 \\
\text { Weight } 0.2 \\
\text { Enabled }\end{array}$ & $\begin{array}{l}\text { Innov } 5 \\
\text { In } 5 \\
\text { Out } 4 \\
\text { Weight } 0.4 \\
\text { Enabled }\end{array}$ & $\begin{array}{l}\text { Innov } 6 \\
\text { In } 1 \\
\text { Out5 } \\
\text { Weight } 0.6 \\
\text { Enabled }\end{array}$ & $\begin{array}{l}\text { Innov } 11 \\
\text { In } 4 \\
\text { Out5 } \\
\text { Weight } 0.6 \\
\text { Enabled }\end{array}$ \\
\hline
\end{tabular}

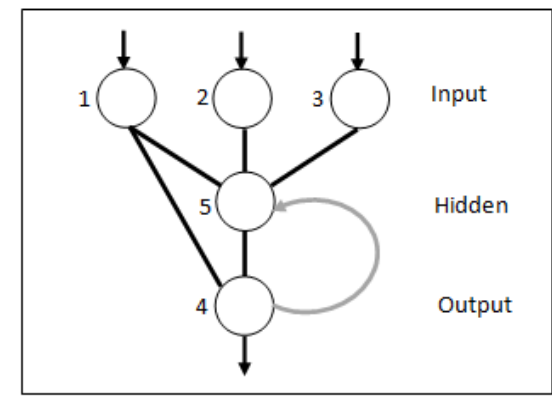

Fig. 3: Demonstration of a NEAT genotype mapping example

\subsection{Backpropagation Neural Network}

The back propagation algorithm is a technique used in developing multilayer neural networks in a supervised manner. The backpropagation algorithm, also known as the error backpropagation algorithm, is based on the errorcorrection learning rule [16]. The algorithm has two passes through the different layers of the network: a forward pass and a backward pass. In the forward pass, an activity pattern is applied to the input nodes of the network, and its effect propagates through the network layer by layer. Finally, a set of outputs is produced as the actual response of the network. During the forward pass the synaptic weights of the networks are all fixed. During the backward pass, the synaptic weights 
are all adjusted in accordance with an error-correction rule. The actual response of the network is subtracted from a desired response to produce an error signal. This error signal is then propagated backward through the network. The synaptic weights are adjusted to make the actual response of the network move closer to the desired response in a statistical sense. The weight adjustment is made according to the generalized delta rule to minimize the error. An example of a multilayer perceptron with two hidden layers is shown in Figure 4.

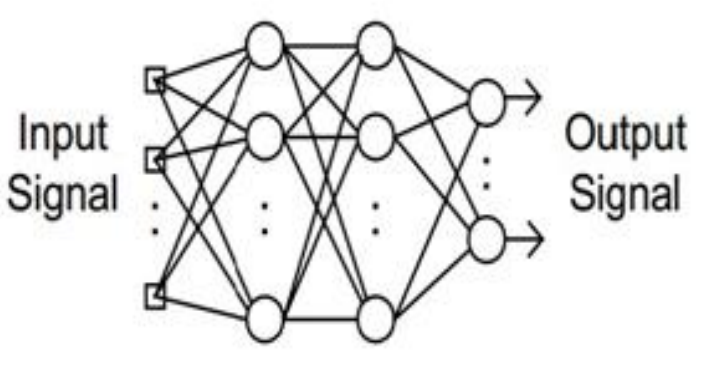

Fig. 4: Multilayer Perceptron with Two Hidden Layers

Two commonly used neuron activation functions for the neuron in Figure 5 are sigmoidal and tansig functions. Both functions are continuously differentiable everywhere and typically has the following mathematical form:

$$
\begin{aligned}
& \text { Sigmoidal } \mathrm{f}(\mathrm{x})=\frac{1}{1+\exp (a x)}, a \succ 0 \\
& \operatorname{tansig} \mathrm{f}(\mathrm{x})=a \tanh (b x), a \& b \succ 0
\end{aligned}
$$
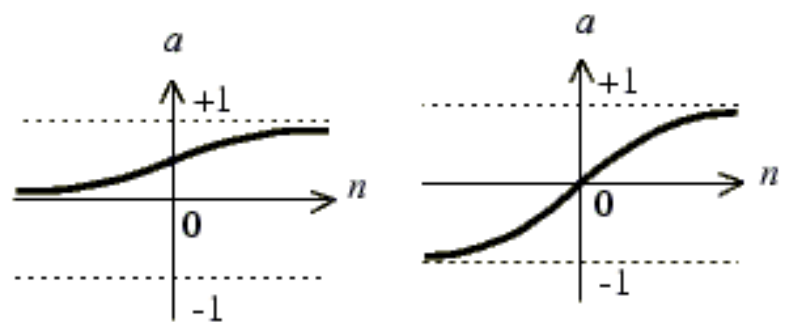

Sigmoidal function

Tansig function

Fig. 5: sigmoidal and Tansig function

\section{EXPERIMENTAL RESULTS}

The NEAT algorithm and standard backpropagation neural network was programmed using Matlab and simulations were performed on the Intel Pentium $42.33 \mathrm{GHz}$ computer. Parameters settings used for the both algorithms after some preliminary experiments is shown in Table 3. These parameters settings control the performance of neural networks. A series of experiments have been run with modified values of mutations probability to determine the influence on the speed of topological evolution.

The performance of the both NEAT and standard neural network is reported in Table 4, where it presents the average test error and standard deviation for both algorithms. The accuracy of NEAT is higher than backpropagation neural network. Moreover, the error of NEAT is smaller than Backpropagation. Table 5 presents a comparison between NEAT algorithm and other algorithms in the literature. It is clear that NEAT algorithm results outperform other algorithms. Moreover, the number of iterations used inside NEAT is near half the number of iterations used for backpropagation, so the execution time is better for NEAT.

It is clear that NEAT algorithm outperform a standard ANN. Both algorithms have been simulated 21 times and the average is evaluated. The NEAT algorithm computational cost is better that standard Neural Network. Figure 6 presents the plot-Box diagram of both algorithms.

Table 3: Parameters Settings

\begin{tabular}{|c|c|c|}
\hline Algorithm & Parameter & Value \\
\hline \multirow{4}{*}{$\begin{array}{c}\text { Standard } \\
\text { Neural } \\
\text { Network }\end{array}$} & Iterations & 1000 \\
\cline { 2 - 3 } & Number of Neurons in Input layer & 13 \\
\cline { 2 - 3 } & Number of Neurons in Hidden layer & $8,5,2$ \\
\cline { 2 - 3 } & Number of Neurons in output layer & 1 \\
\hline \multirow{4}{*}{$\begin{array}{c}\text { NEAT } \\
\text { Algorithm }\end{array}$} & Iterations & 1000 \\
\cline { 2 - 3 } & Population size & 50 \\
\cline { 2 - 3 } & Number of Input nodes & 2 \\
\cline { 2 - 3 } & Number of output nodes & 1 \\
\cline { 2 - 3 } & Mutate Add link Prob. & 0.5 \\
\hline
\end{tabular}

Table 4: Average test accuracy (Smallest Training Error)

\begin{tabular}{|c|c|}
\hline Algorithm & $\begin{array}{c}\text { Avg. Test Accuracy and } \\
\text { standard Deviation }\end{array}$ \\
\hline NEAT Algorithm & $98.5 \pm 0.004$ \\
\hline Standard Neural Network & $95.3 \pm 1.425$ \\
\hline
\end{tabular}

Table 5: comparison between algorithms in literature

\begin{tabular}{|l|c|c|}
\hline \multicolumn{1}{|c|}{ Algorithm } & Accuracy & $\begin{array}{c}\text { Number of } \\
\text { Iterations }\end{array}$ \\
\hline NEAT Algorithm & $98.5 \%$ & 6854 \\
\hline Standard Neural Network & $95.3 \%$ & 15202 \\
\hline Fogel et al [8] & $98.1 \%$ & 5100 \\
\hline Back- Prop of Abbass et al. [20] & $97.5 \%$ & 10000 \\
\hline
\end{tabular}
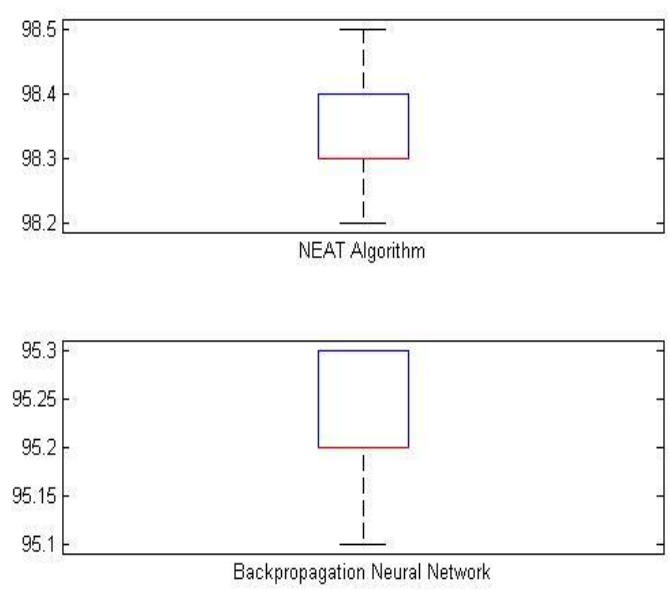

Fig. 6: Plot-Box diagram for NEAT and standard neural network 


\section{CONCLUSION AND FUTURE WORK}

In this paper, we compare the performance between NEAT algorithm and standard neural network to predict breast cancer. NEAT is attempting to evolve optimal or near-optimal neural network topologies to solve problems. Thus, a very good approach to evolving a neural network to solve a given problem seems to be to start with relatively minimal architectures while including both complexifying and simplifying mutations, their rates balanced in such a way to produce an even search in both directions through the range of possible architectures. This approach should yield both more efficient searches and more efficient solutions than either complexifying or simplifying algorithms alone. However, work remains to be done to verify these conclusions across a wide range of domains of varying difficulty. The experiments in this paper have demonstrated that while complexifying and simplifying dynamics are both important in the search for optimal neural network topologies, these dynamics work better together than either dynamic working alone.

\section{REFERENCES}

[1] C.E. Floyd, J.Y. Lo, A.J. Yun, D.C. Sullivan, and P.J. Kornguth. Prediction of breast cancer malignancy using an artificial neural network. Cancer, 74:2944-2998, 1994.

[2] D.B. Fogel, E.C. Wasson, and E.M. Boughton. Evolving neural networks for detecting breast cancer. Cancer letters, 96(1):49-53, 1995.

[3] D. Furundzic, M. Djordjevic, and A.J. Bekic. Neural networks approach to early breast cancer detection. Systems Architecture, 44:617-633, 1998.

[4] D.E. Rumelhart, G.E. Hinton, and R.J. Williams. Learning internal representations by error propagation. In J.L. McClelland D.E. Rumelhart and the PDP Research Group Eds, editors, Parallel Distributed Processing: Explorations in the Microstructure of Cognition., Foundations, 1, MIT Press, Cambridge, pages 318-362, 1986

[5] Y.Z. Wu, M.L. Giger, K. Doi, C.J. Vyborny, R.A. Schmidt, and C.E. Metz. Artificial neural networks in mammography: application to decision making in the diagnosis of breast cancer. Radiology, 187:81-87, 1993.

[6] P. Wilding, M.A. Morgan, A.E. Grygotis, M.A. Shoffner, and E.F. Rosato. Application of backpropagation neural networks to diagnosis of breast and ovarian cancer. Cancer Letter, 77:145-153, 1994

[7] C.E. Floyd, J.Y. Lo, A.J. Yun, D.C. Sullivan, and P.J. Kornguth. Prediction of breast cancer malignancy using an artificial neural network. Cancer, 74:2944-2998, 1994.

[8] D.B. Fogel, E.C. Wasson, and E.M. Boughton. Evolving neural networks for detecting breast cancer. Cancer letters, 96(1):49-53, 1995.

[9] R. Setiono. Extracting rules from pruned neural networks for breast cancer diagnosis. Artificial Intelligence in Medicine, 8:37-51, 1996.

[10] R. Setiono. Generating concise and accurate classification rules for breast cancer diagnosis. Artificial Intelligence in Medicine, 18:205-219, 2000.

[11] P.C. Pendharkar, J.A. Rodger, G.J. Yaverbaum, N. Herman, and M. Benner. Association, statistical, mathematical and neural approaches for mining breast cancer patterns. Expert Systems with Applications, 17:223-232, 1999

[12] D. Furundzic, M. Djordjevic, and A.J. Bekic. Neural networks approach to early breast cancer detection. Systems Architecture, 44:617-633, 1998.

[13] P.C. Jong, H.H. Tae, W.P. Rae, A Hybrid Bayesian Network Model for Predicting Breast Cancer Prognosis, J Kor Soc Med Informatics, 15(1):49-57, 2009.

[14] Y.Z. Wu, M.L. Giger, K. Doi, C.J. Vyborny, R.A Schmidt, and C.E. Metz. Artificial neural networks in mammography: application to decision making in the diagnosis of breast cancer. Radiology, 187:81-87, 1993.

[15] P. Pornchai and B. Pleumjit. Color Based Segmentation of Nuclear Stained Breast Cancer Cell Images. IEEE transactions on electrical eng., electronics, and communications, 5(2), 2007.

[16] F. Amato , A. López, E.M. Peña-Méndez , P.Vaňhara , A. Hamp and J.Havel. Artificial neural networks in medical diagnosis. Journal of APPLIED BIOMEDICINE, 11: 47-58, 2013.

[17] O. K. Stanley and R. Miikkulainen. Evolving Neural Networks through Augmenting Topologies. Evolutionary Computation, Vol. 10, No. 2 , 99-127,2002. 